\title{
Sitio Web y aplicación móvil para el control de datos climatológicos a tráves de sensores en un prototipo de estación agrometeorológica
}

\section{Web Site and mobile application for the control of climatological data through sensors in a prototype of agrometeorological station}

\author{
DE LEÓN-CASTREJÓN, Andrés $\dagger^{*}$, MELQUIADES-JIMÉNEZ, José Uriel y NORIEGA-CANTÚ, \\ David Heriberto
}

Universidad Tecnológica de la Región Norte de Guerrero

ID $1^{\mathrm{er}}$ Autor: Andrés, De León-Castrejón / ORC ID: 0000-0003-2864-2358, CVU CONACYT ID: 744847

ID $1^{\text {er }}$ Coautor: José Uriel, Melquiades-Jiménez / ORC ID: 0000-0001-9690-292X, CVU CONACYT ID: 1000162

ID $2^{\text {do }}$ Coautor: David Heriberto, Noriega-Cantú / ORC ID: 0000-0002-8215-4104, CVU CONACYT ID: 74588

DOI: $10.35429 /$ JCA.2019.12.3.8.17

Recibido Julio 30, 2019; Aceptado Noviembre 30, 2019

\begin{abstract}
Resumen
El Instituto Nacional de Investigaciones Forestales, Agrícolas y Pecuarias, Campo Experimental Iguala y la Universidad Tecnológica de la Región Norte de Guerrero, están uniendo sus conocimientos en el ámbito tanto científico como tecnológico con el propósito de desarrollar proyectos de colaboración. En el Estado de Guerrero hay una escasa información agroclimática veraz y oportuna que sirva como factor determinante en la toma de decisiones en los procesos productivos. El presente estudio tiene como finalidad el de desarrollar un sitio web y una aplicación móvil, donde se tenga acceso a la información emitida por el prototipo de estación agrometeorológica. Los datos serán ingresados a una base de datos en el sitio web y serán almacenados cada 10,15 o 30 minutos de las variables de clima, tales como: temperatura, humedad relativa, precipitación, radiación solar, humedad de la hoja, velocidad y dirección del viento. La aplicación móvil permitirá consultar los datos climatológicos históricos y en tiempo real. Esto resultará en un mejor control de la información del medio ambiente con el fin de que los productores en el cultivo de maíz, frutales y hortalizas puedan prevenir y evitar las plagas y enfermedades de sus siembras.
\end{abstract}

Sitio Web, Aplicación Móvil, Datos Climatológicos

\begin{abstract}
The National Institute of Forestry, Agriculture and Livestock Research, Iguala Experimental Field and the Technological University of the North Region of Guerrero, are joining their knowledge in the scientific and technological field with the purpose of developing collaborative projects. In the State of Guerrero, there is a lack of accurate and timely agroclimatic information that serves as a determining factor in decision-making in productive processes. The purpose of this study is to develop a website and a mobile application, where the information issued by the prototype of agrometeorological station can be accessed. The data will be entered into a database on the website and will be stored every 10,15 or 30 minutes of the climate variables, such as: temperature, relative humidity, precipitation, solar radiation, leaf moisture, speed and direction of the wind. The mobile application will allow to consult historical and real-time weather data. This will result in better control of environmental information so that producers in the cultivation of corn, fruit and vegetables can prevent and avoid pests and diseases of their crops.
\end{abstract}

Website, Mobile Application, Climatological Data

Citación: DE LEÓN-CASTREJÓN, Andrés, MELQUIADES-JIMÉNEZ, José Uriel y NORIEGA-CANTÚ, David Heriberto. Sitio Web y aplicación móvil para el control de datos climatológicos a tráves de sensores en un prototipo de estación agrometeorológica. Revista de Cómputo Aplicado. 2019, 3-12: 8-17

\footnotetext{
* Correspondencia al Autor (Correo electrónico: andresdlc@utrng.edu.mx)

$\dagger$ Investigador contribuyendo como primer Autor.
} 


\section{Introducción}

El presente estudio tiene como propósito el desarrollar e implementar un sitio web para alojarlo en un hosting y una aplicación móvil, ambas aplicaciones realicen conexión hacia y desde un sistema embebido que se configurará en un prototipo de estación agrometeorológica.

El prototipo de la estación agrometeorológica realizará el sensado de variables climatológicas como son la temperatura ambiental, precipitación pluvial, humedad relativa, radiación solar y ultravioleta, dirección y velocidad del viento a través de un api restful service para comunicarse con el sitio web, para que éste realice el registro de los datos sensados en su base de datos y éstos sean procesados para obtener información que permita la toma de decisiones a la hora de sembrar maíz en época de temporal.

Asi mismo, la aplicación móvil permita realizar la consulta en tiempo real de los datos que este sensando en un momento dado el prototipo de la estación agrometeorológica y pueda acceder también a los datos históricos que ya se encuentren guardados en el sitio web.

\section{Problema}

El Instituto Nacional de Investigaciones Forestales, Agrícolas y Pecuarias del Estado de Guerrero (INIFAP), Campo Experimental Iguala, ubicado en la localidad de Tuxpan, Gro., tiene bajo su control poco más de 20 estaciones climatológicas ubicadas en las siete regiones del Estado de Guerrero, las cuales permiten leer variables a través de sensores, tales como: temperatura ambiental, humedad relativa, precipitación pluvial, radiación solar y ultravioleta, velocidad y dirección del viento ${ }^{[3]}$.

De las más de 20 estaciones climatológicas únicamente funcionan $3, \mathrm{y}$ debido a la ubicación que tiene cada estación en algún lugar de la región del Estado de Guerrero, se torna complicada la forma de comunicación con ellas, ya que éstas, se encargan de leer las variables del medio ambiente, por lo que su mantenimiento físico y deterioro de los componentes electrónicos y eléctricos de que están compuestas se torna complicado.
Ahora bien, de las 3 estaciones ubicadas en distintos lugares del Estado de Guerrero, 2 están instaladas en el campus experimental de Iguala, Gro; y de éstas, solo 1 alcanza el 75\% de su funcionalidad.

\section{Justificación}

Como ya se mencionó antes, se tienen 3 estaciones agrometeorológicas, 2 de las cuales ya no funcionan para sensar las variables climatológicas, por causa del deterioro de sus elementos electrónicos y a la falta de un mantenimiento periódico.

Por otro lado, de manera paralela se está desarrollando un prototipo de estación agrometeorológica que va a permitir la lectura de las variables climatológicas, las cuales podrán ser almacenadas en el sitio web, con el propósito de obtener datos climatológicos diarios e irse almacenando posteriormente para contar con datos históricos.

Una vez que la información sea controlada en la base de datos a través de un sitio web y ésta a su vez pueda ser consultada a través de una aplicación móvil, todo ello coadyuvará en la toma de decisiones para mejorar la siembra de maíz de temporal.

\section{Hipotésis}

Con el desarrollo e implementación de un sitio web y una aplicación móvil se estará en condiciones de recibir los datos de las variables climatológicas sensadas a través de un prototipo de estación agrometeorológica para apoyar en la toma de decisiones respecto de la siembra de granos, sanidad agrícola y frutales en el temporal de lluvias.

\section{Objetivos}

\section{Objetivo General}

Desarrollar e implementar un sitio web y una aplicación móvil para el control de datos climatológicos a través de sensores en un prototipo de estación agrometeorológica, que apoye a la toma de decisiones para la siembra de granos, sanidad agrícola y frutales en el temporal de lluvias. 


\section{Objetivos Específicos}

- Construir la base de datos eclima que incluya las tablas, tales como: contacto, directorio, estaciones, estacion_agrometeorologica, estados, login, municipios, posts, tbl_tokens y usuario.

- Diseñar interfaces gráficas de usuario como formularios web, tales como: el login para sesiones de usuarios y administrador, datos climatológicos, estaciones, mapas, datos diarios, publicaciones, artículos científicos, folletos técnicos, directorio, inifap guerrero y email.

- Desarrollar los módulos en php para el control de información y procesamiento en el sitio web.

- Diseñar una interfaz gráfica de usuario, mostrando un mapa, con ubicaciones específicas para actualizar, eliminar y agregar cada estación agrometeorológica posicionándolas en algún punto geográfico del estado de guerrero.

- Diseñar las interfaces gráficas para la aplicación móvil que incluya las opciones como el sitio web para consultar los datos diarios e históricos de las variables climatológicas de manera remota.

- Contratar un hosting y crear el dominio datoseclima. opds. website para alojar el sitio web y la base de datos del proyecto para realizar pruebas.

- Implementar dos apis restful (microservicios), uno para conectar la base de datos del sitio web con la tarjeta de adquisición de datos xatellite y el otro para conectar la aplicación móvil con el sitio web de forma remota.

\section{Marco Teórico}

En esta sección se mencionarán de manera breve las herramientas que serán utilizadas para desarrollar el proyecto, tales como:

Php. Es un lenguaje de programación que se utilizará para construir nuestro sitio web, así también se puede incrustar en html para lograr mejor dinamismo, por otra parte, puede conectar la base de datos del sitio. ${ }^{[13]}$
Enterprise Architect. Es una herramienta de diseño y análisis uml, cubriendo el análisis, diseño y desarrollo de software desde el paso de los requerimientos a través de las etapas del análisis, modelos de diseño, pruebas y mantenimiento. Se ocupará en nuestro proyecto para modelar el sitio web a través de diagramas como: casos de uso, secuencias y clases.

Xampp. Es un software que integra en una sola aplicación un servidor web como apache, interprete de php, servidor de base de datos para mysql, en nuestro caso se ocupará para construir la base de datos e interpretación del código en php de nuestras interfaces gráficas del sitio web.

Html5. Es un lenguaje que sirve para colocar etiquetas o marcas en un texto que indique como debe verse, es decir es un sistema de etiquetas. Para nuestro proyecto se utilizará combinándolo con php para mejorar el diseño y desarrollo de las interfaces gráficas.

Dreamweaver. Es un software para crear sitios web, aquí se construirán los formularios web, menú de opciones y demás elementos que ofrezcan una interfaz más atractiva y fácil de usar por los usuarios que utilicen el sitio web.

Notepad++. Es un editor de texto y editor de código fuente, se ocupará para escribir los códigos en php de los módulos que contemplará nuestro sitio web.

JavaScript. Es un lenguaje de programación interpretado, será ocupado para realizar mejoras en las interfaces gráficas de nuestro sitio web y los formularios. Con este lenguaje se podrán implementar documentos pdf que ocupará el sitio web.

Android sdk. Es un kit de desarrollo de software para el lenguaje java, en él será desarrollada la aplicación móvil y depurarla a través de un emulador o directamente en un dispositivo móvil, a través de Android Studio.

Bootstrap. Será ocupado para desarrollar las interfaces gráficas del sitio web y la aplicación móvil, de tal forma que sean vistas en el display de cualquier dispositivo, donde se muestre. 
El Dr. Arturo A. Pacheco Espejel establece que "la investigación tecnológica es la que se encarga de desarrollar un conocimiento enfocado a diseñar o mejorar los instrumentos y las herramientas que el hombre necesita para potenciar su fuerza de trabajo y sus capacidades transformadoras de la realidad". [7]

\section{Metodología de Desarrollo de Software}

Así mismo el software se ha convertido en el elemento clave de la evolución de los sistemas y productos basados en computadoras, así como en una de las tecnologías más importantes en el ámbito mundial.

En la actualidad el software evoluciona de acuerdo con un conjunto de leyes que han permanecido inalteradas a lo largo de 30 años.

La intención de la ingeniería de software es proporcionar un marco general para construir software con una calidad mucho mayor. ${ }^{[8]}$

Para la construcción del sitio web y la aplicación móvil se utilizó el modelo en cascada o clásico, tal como se describe en la tabla 1.

\section{Metodología a desarrollar}

\begin{tabular}{|c|c|}
\hline Etapa & Descripción \\
\hline $\begin{array}{l}\text { Recopilación de } \\
\text { la Información }\end{array}$ & $\begin{array}{l}\text { En esta etapa se recolectará } \\
\text { información acudiendo al Instituto } \\
\text { Nacional de Investigaciones } \\
\text { Forestales Agrícolas y Pecuaria } \\
\text { Iguala. A través de una entrevista al } \\
\text { Dr. David H. Noriega Cantú } \\
\text { investigador en el área de Sanidad } \\
\text { Agrícola y Frutales. }\end{array}$ \\
\hline Análisis / diseño & $\begin{array}{l}\text { De la información obtenida, por la } \\
\text { entrevista, se analizarán los } \\
\text { requerimientos funcionales para } \\
\text { desarrollar el sitio web y la } \\
\text { aplicación móvil. }\end{array}$ \\
\hline Codificación & $\begin{array}{l}\text { Una vez que se concluya el análisis } \\
\text { y el diseño se procederá a codificar } \\
\text { el sitio web y la aplicación móvil. }\end{array}$ \\
\hline Pruebas & $\begin{array}{l}\text { En esta fase se realizarán las } \\
\text { pruebas necesarias al sitio web y la } \\
\text { aplicación móvil para la detección } \\
\text { de los posibles errores que una vez } \\
\text { detectados se dará solución a cada } \\
\text { uno de ellos. }\end{array}$ \\
\hline Documentación & $\begin{array}{l}\text { En esta fase se documentará el } \\
\text { proyecto para consulta de los } \\
\text { usuarios. }\end{array}$ \\
\hline
\end{tabular}

Tabla 1 Metodología a desarrollar Fuente: Elaboración Propia

\section{Diseño y Desarrollo}

Un diagrama es un gráfico que presenta en forma esquematizada información relativa e inherente a algún tipo de ámbito, uml está compuesto por diversos elementos gráficos que se combinan para conformar diagramas. Debido a que uml es un lenguaje, cuenta con reglas para combinar tales elementos. La finalidad de los diagramas es presentar diversas perspectivas de un sistema, a las cuales se les conoce como modelo ${ }^{[4]}$. Para ello se explicarán los diagramas de casos de uso y de clases que reflejan la estructura estática del sitio web.

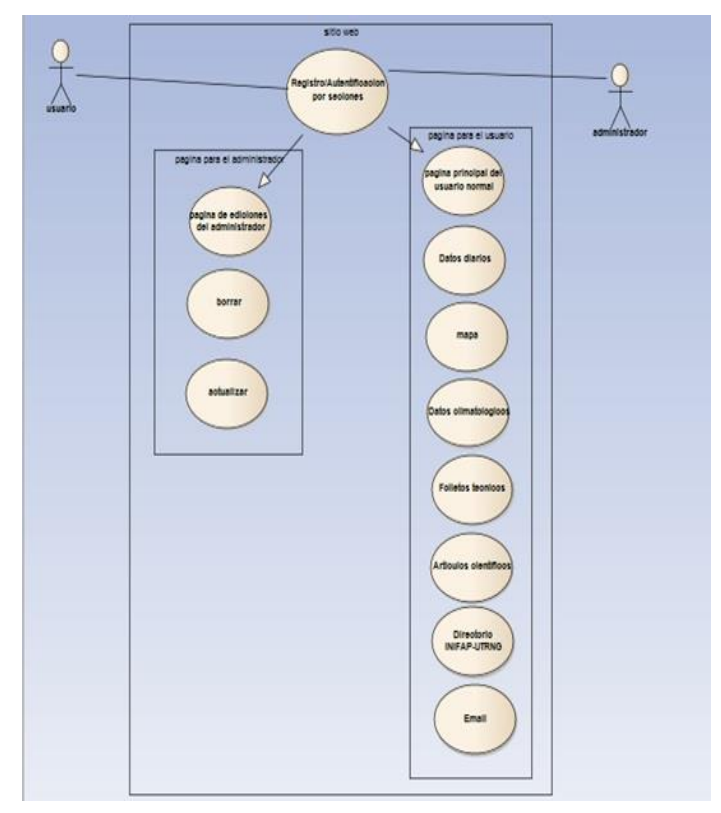

Figura 1 Diagrama de casos de uso principal Fuente: Elaboración Propia

El siguiente diagrama modela el registro del usuario para que capture la información que se le pide llenar, ver figura 2.

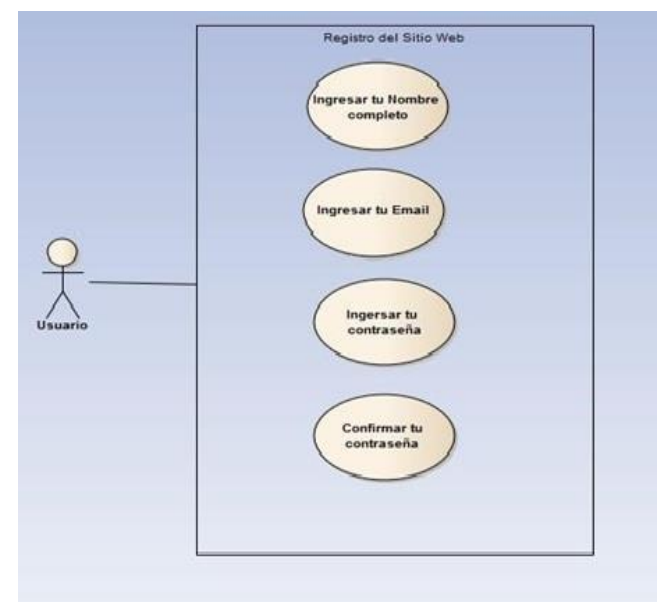

Figura 2 Diagrama de casos de uso Registrar usuario Fuente: Elaboración Propia

En este diagrama se modela la forma de autenticar un usuario para acceder al sitio web. DE LEÓN-CASTREJÓN, Andrés, MELQUIADES-JIMÉNEZ, José Uriel y NORIEGA-CANTÚ, David Heriberto. Sitio Web y aplicación móvil para el control de datos climatológicos a tráves de sensores en un prototipo de estación agrometeorológica. Revista de Cómputo Aplicado. 2019. 


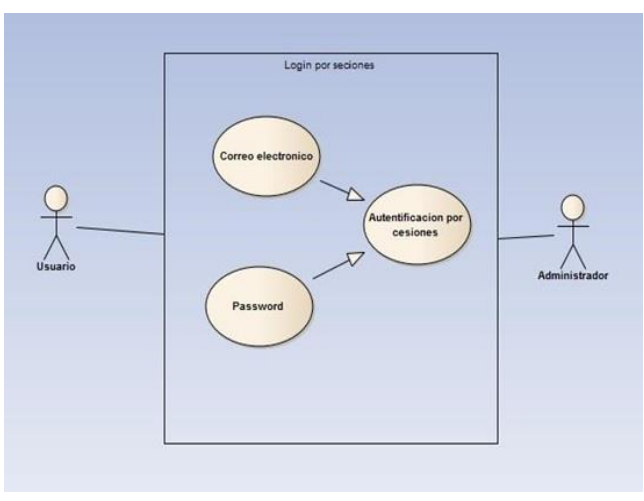

Figura 3 Diagrama de casos de uso login por sesiones de usuario

Fuente: Elaboración Propia

A continuación, se modelan las actividades que tiene un usuario administrador del sitio web y que le son inherentes de acuerdo al rol de usuario.

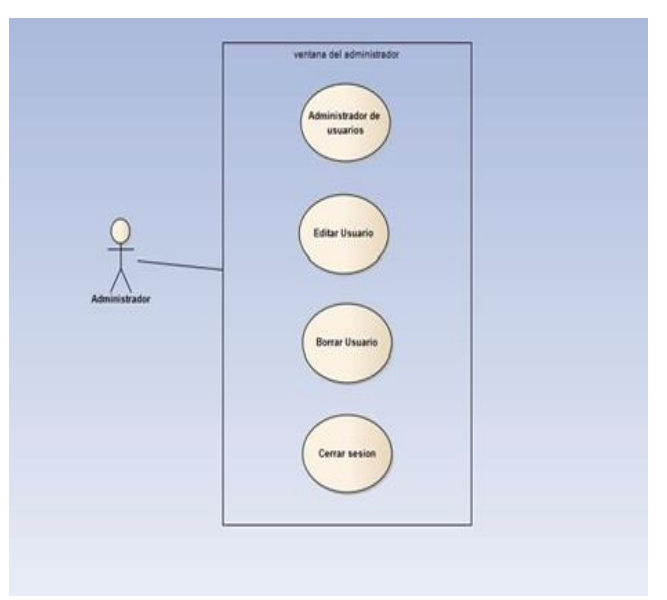

Figura 4 Diagrama de casos de uso vista de administrador Fuente: Elaboración Propia

En el siguiente diagrama se modelan las clases que contendrá el sitio web como parte de la estructura estática del mismo.

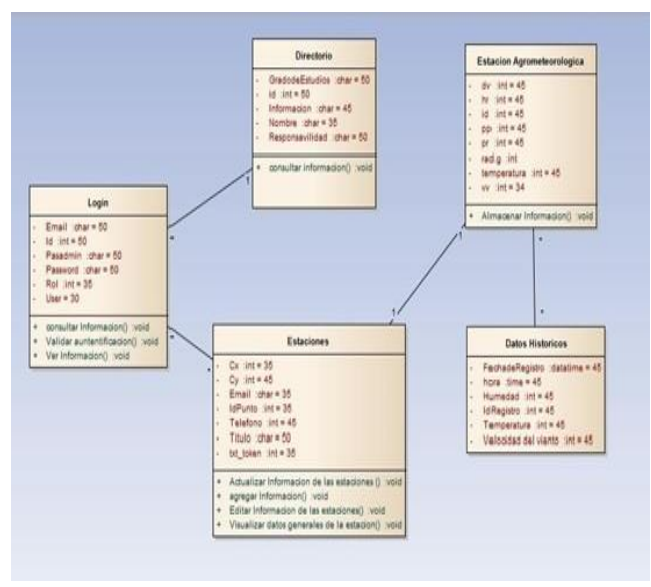

Figura 5 Diagrama de clases del sitio web Fuente: Elaboración Propia

\section{Modelo relacional de la base de datos}

En la siguiente figura, se muestra el modelo relacional de la base de datos, con base en el manejador mysql a través de phpMyAdmin.

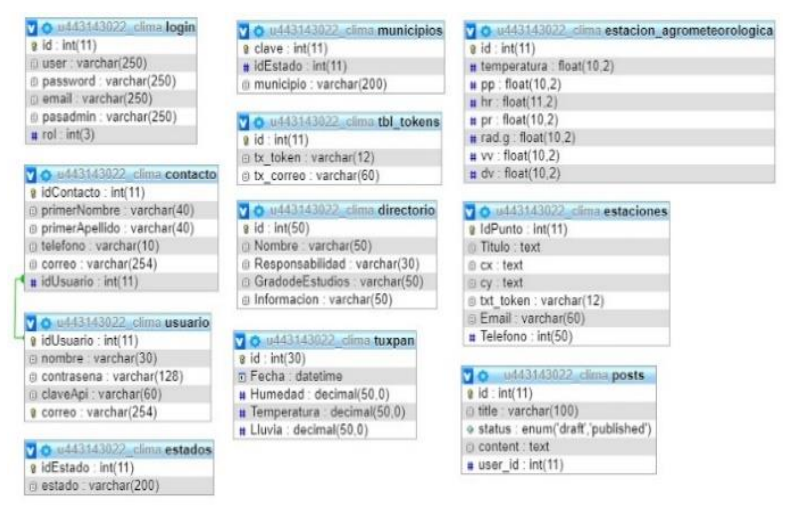

Figura 6 Modelo relacional de la base de datos Fuente: Elaboración Propia

\section{Resultados}

Una vez presentado el análisis, diseño y desarrollo del proyecto, ahora se describirán los resultados obtenidos con el sitio web y la aplicación móvil y son las siguientes:

Del sitio web se puede mostrar un login de usuario para el acceso al menú principal o en su defecto en la parte inferior el registro por si fuese un usuario nuevo, tal como se observa en la figura 7.

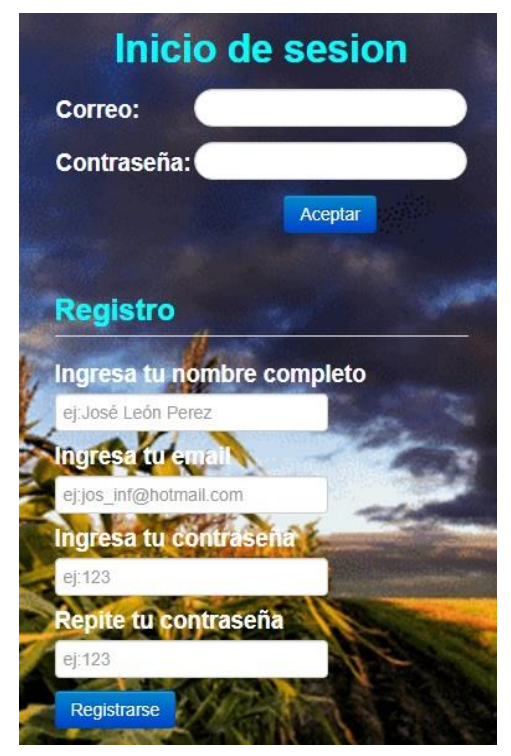

Figura 7 Inicio de sesión de usuario

Fuente: http://datoseclima.opds.website/

Ya que el usuario se autentificó con su nombre de usuario que es su email y una contraseña, estará en condiciones de entrar al menú principal a navegar en las diferentes secciones de menú tales como:

DE LEÓN-CASTREJÓN, Andrés, MELQUIADES-JIMÉNEZ, José Uriel y NORIEGA-CANTÚ, David Heriberto. Sitio Web y aplicación móvil para el control de datos climatológicos a tráves de sensores en un prototipo de estación agrometeorológica. Revista de Cómputo Aplicado. 2019. 
Datos climatológicos, estaciones, publicaciones, directorio, opciones y cerrar sesión, como se observa en la figura 8.

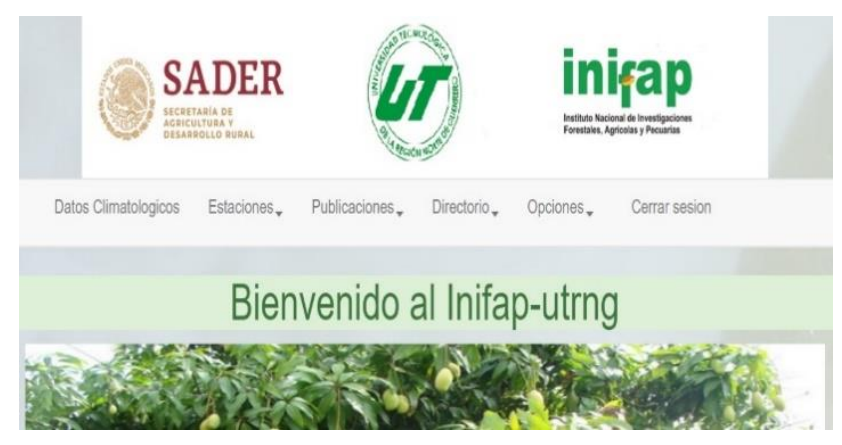

Figura 8 Vista del menú principal del sitio web Fuente: http://datoseclima.opds.website/InicioInifap\%20$\% 20$ copia.html

Una vez que ingresa el usuario se puede navegar en las diferentes secciones que le proporciona el sitio web, en este caso se muestra la sección Datos Climatológicos. Ver figura 9.

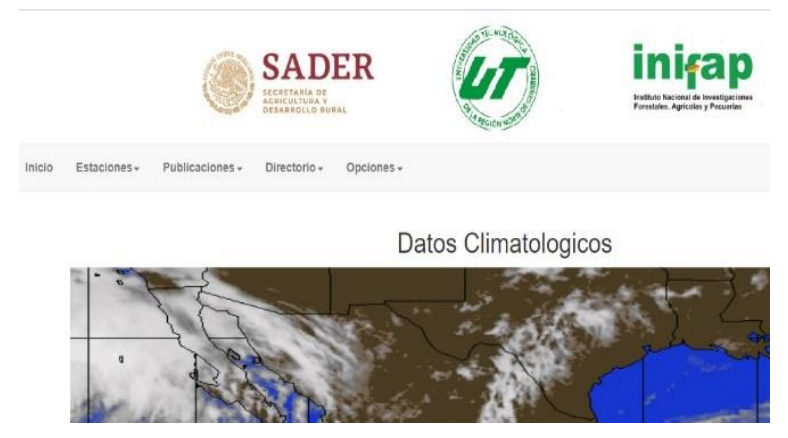

Figura 9 Vista de datos climatológicos (parte 1) Fuente:

http://datoseclima.opds.website/pronosticoestacional.htm $l$

En esta segunda parte de la vista de datos climatológicos se pueden ver los registros organizado por periodos de la información que sensa el prototipo de la estación agrometeorológica que está programado para cada 10, 15 o 30 minutos, estos datos se conocen como datos históricos, ver figura 10.

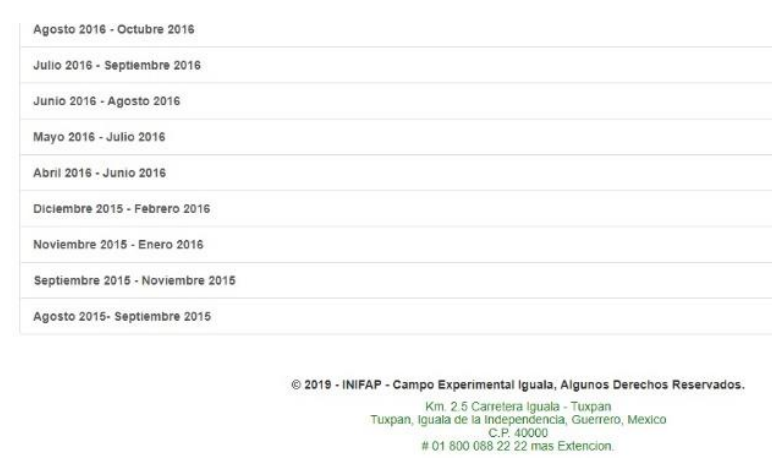

Figura 10 Vista de datos climatológicos (parte 2)

Fuente:

http://datoseclima.opds.website/pronosticoestacional.htm $l$

ECORFAN $^{\circledR}$ Todos los derechos reservados
En la figura 11, se muestra el mapa que depende de la sección Estaciones donde se muestra un mapa de google map, se permite dar de alta y ubicar geográficamente una estación agrometeorológica guardando sus datos de localización como son latitud y longitud para que posteriormente pueda realizar el sensado de variables climatológicas de acuerdo en donde se coloque.

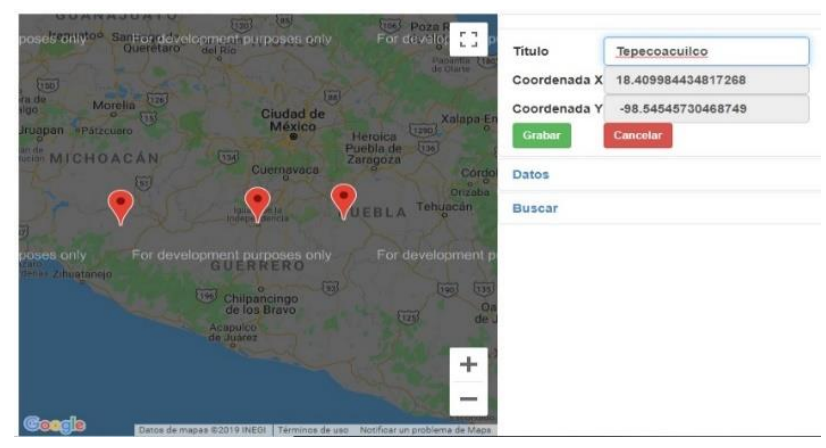

Figura 11 Vista del mapa de google de la sección Estaciones

Fuente: http://maps.googleapis.com/maps-api-v3/api/js

En la figura 12, se muestra la sección de datos diarios, en ésta se pueden observar las diferentes abreviaturas que tienen las variables climatológicas que sensa el prototipo de estación agrometeorológica.

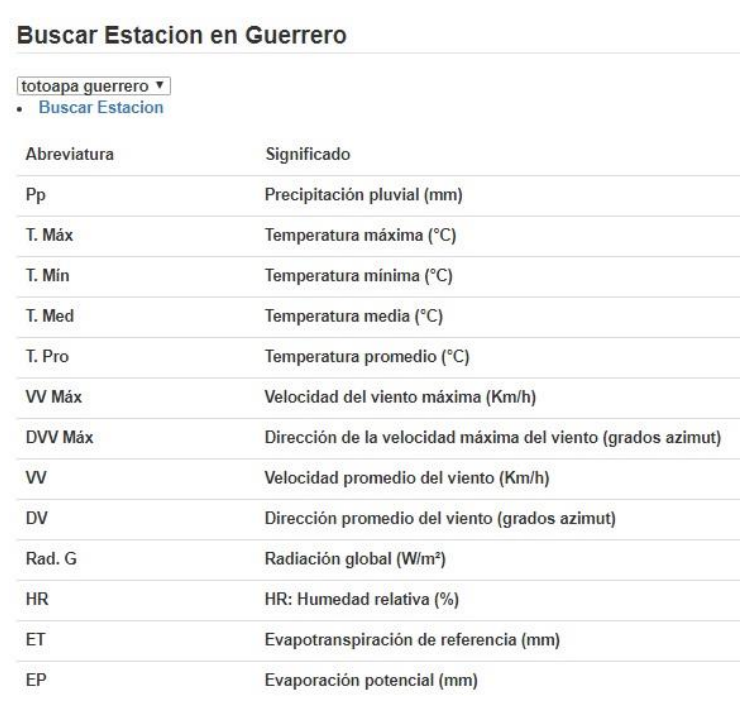

Figura 12 Vista de datos diarios

Fuente: http://datoseclima.opds.website/datos.php

Además, se puede observar en la parte superior izquierda una lista de estaciones agrometeorológicas, y una vez que se elige una de ellas se mostrarán unos controles de la plataforma ubidots.com para que se realicen pruebas de adquisición de datos.

DE LEÓN-CASTREJÓN, Andrés, MELQUIADES-JIMÉNEZ, José Uriel y NORIEGA-CANTÚ, David Heriberto. Sitio Web y aplicación móvil para el control de datos climatológicos a tráves de sensores en un prototipo de estación agrometeorológica. Revista de Cómputo Aplicado. 2019. 


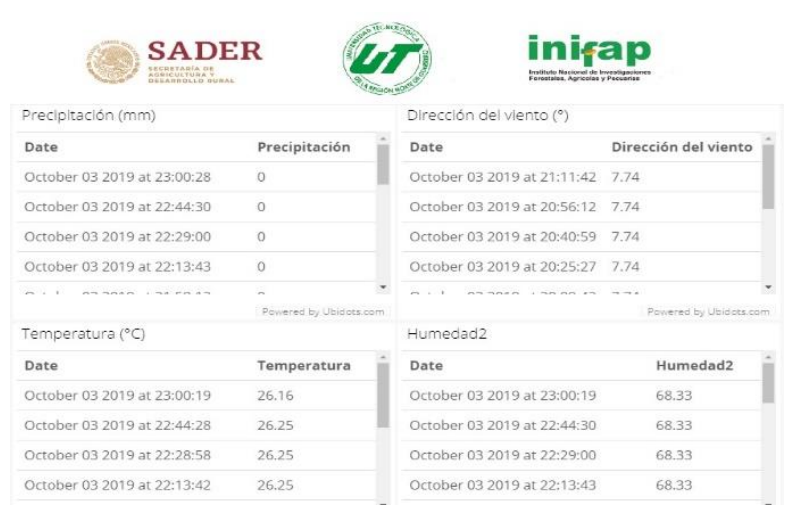

Figura 13 Datos climatológicos de los sensores utilizando controles ubidots (parte 1)

Fuente: $\quad$ http://datoseclima.opds.website/PHPExcel1.8/mileer.php

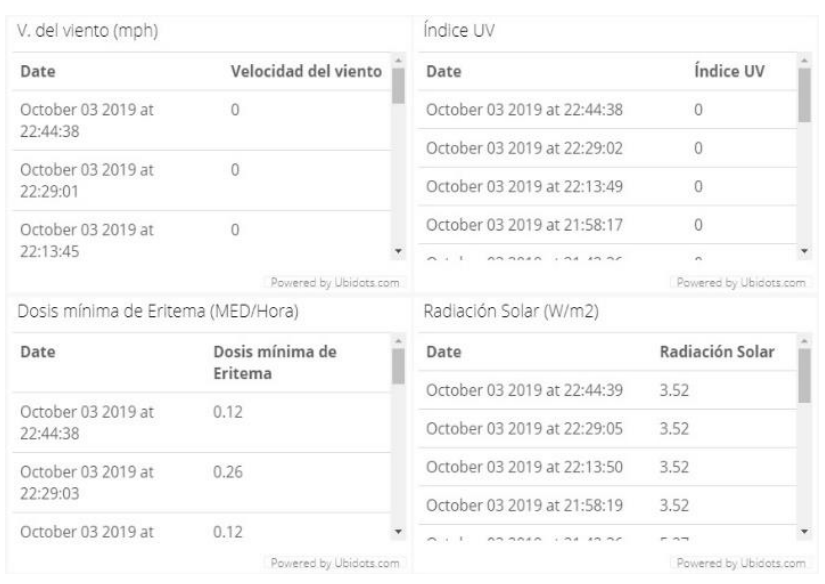

Figura 14 Datos climatológicos de los sensores utilizando controles ubidots (parte 2)

Fuente: $\quad$ http://datoseclima.opds.website/PHPExcel1.8/mileer.php

En otro orden de ideas, se elige la única estación agrometeorológica de la lista que ya se encuentre dada de alta, se muestra una interfaz gráfica que hace conexión con la plataforma de ubidots.com en la cual a través de un api service, ubidots.com se comunica con la tarjeta de adquisición de datos xatellite y los visualiza en un formulario web. Posteriormente se pretende que con el api restful service que se implemente tanto en el sitio web como en la aplicación móvil se almacene la información de las variables climatológicas directamente al sitio web.

Con respecto de la sección Publicaciones, ésta tendrá dos apartados: uno es para mostrar los artículos científicos que desarrollan los investigadores del inifap y que pueden subir para que los puedan ver otros investigadores interesados, tal como se observa en la figura 15, el otro apartado es para la publicación de folletos técnicos, tal como se muestra en la figura 16, aquí de manera similar a los artículos científicos, los folletos también se podrán consultar y descargar.

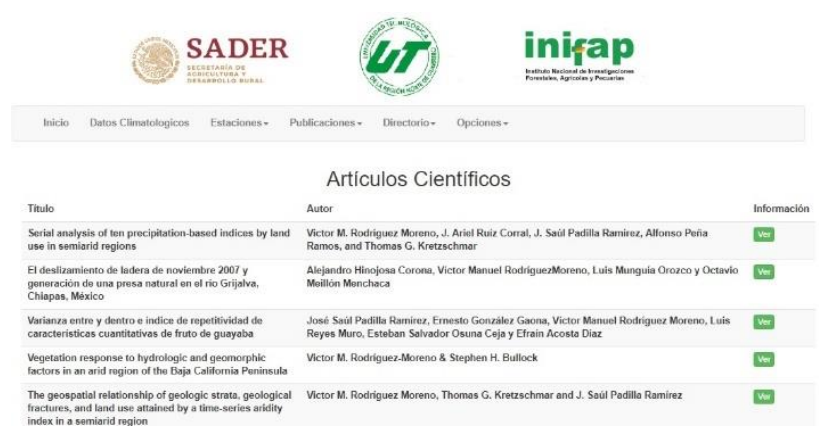

Figura 15 Vista de artículos científicos Fuente:

http://datoseclima.opds.website/articulosientificos.html

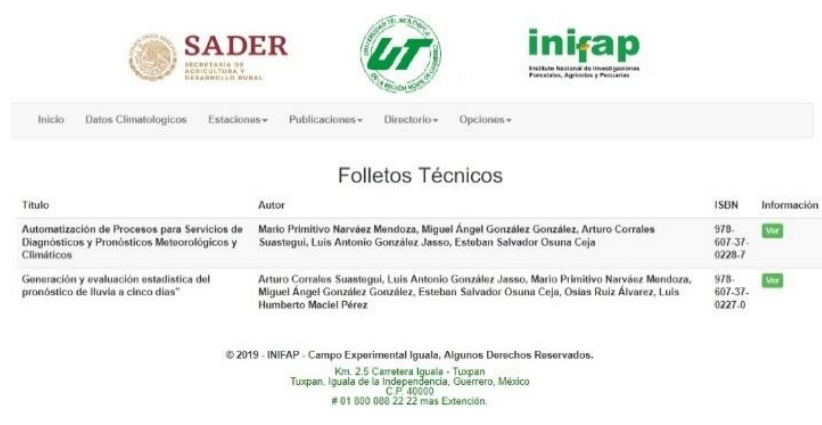

Figura 16 Vista de folletos técnicos

Fuente:

http://datoseclima.opds.website/folletotecnico.html

Continuando con las secciones del menú principal se tiene el apartado Directorio, en éste se muestra la vista Inifap Guerrero, en la cual se observa una lista de investigadores del inifap desde el director hasta el último investigador que realiza proyectos en su área específica, tal como se muestra en la figura 17.

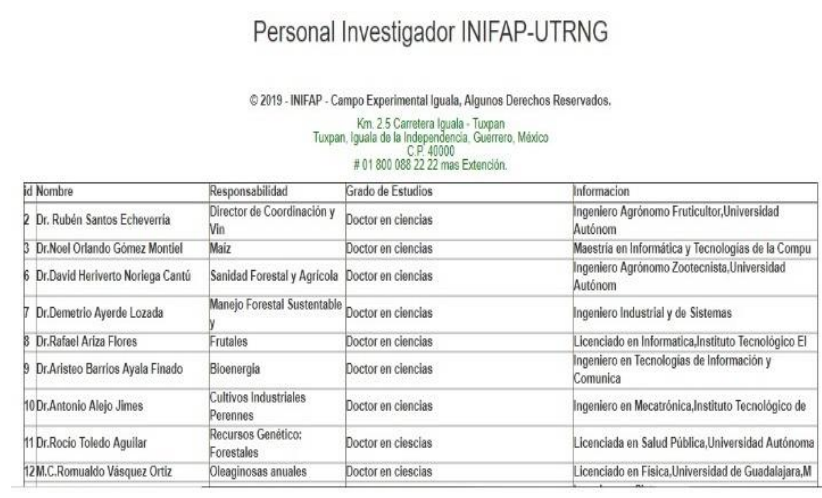

Figura 17 Vista inifap guerrero

Fuente: http://datoseclima.opds.website/prueva.php

Inclusive este apartado almacena una lista en una tabla de base de datos y tiene la característica que se puede actualizar, agregar o eliminar algún registro de acuerdo si pertenece o ya no el investigador al inifap guerrero.

DE LEÓN-CASTREJÓN, Andrés, MELQUIADES-JIMÉNEZ, José Uriel y NORIEGA-CANTÚ, David Heriberto. Sitio Web y aplicación móvil para el control de datos climatológicos a tráves de sensores en un prototipo de estación agrometeorológica. Revista de Cómputo Aplicado. 2019. 
Por otro lado, en la sección de Opciones se tiene la opción de email, la cual muestra un formulario en Outlook para que el usuario pueda ingresar a su cuenta de correo si estuviese registrado en dicho servicio, tal como se observa en la figura 18.

\section{Outlook Web App}

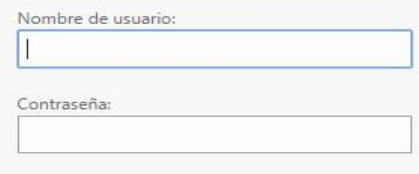

$\Theta$ iniciar sesión

Figura 18 Vista Opciones (email)

Fuente:

https://mail.inifap.gob.mx/owa/auth/logon.aspx?replace Current $=1 \&$ url=https $\% 3$ a\% 2 f\% 2 fmail.inifap.gob.mx\% $2 f$ owa siguiente:

De la aplicación móvil se tiene lo En la figura 19, se observa la vista del inicio de sesion de la aplicación móvil donde el usuario debe capturar un nombre de usuario y una contraseña, para ingresar al menú principal de la misma.

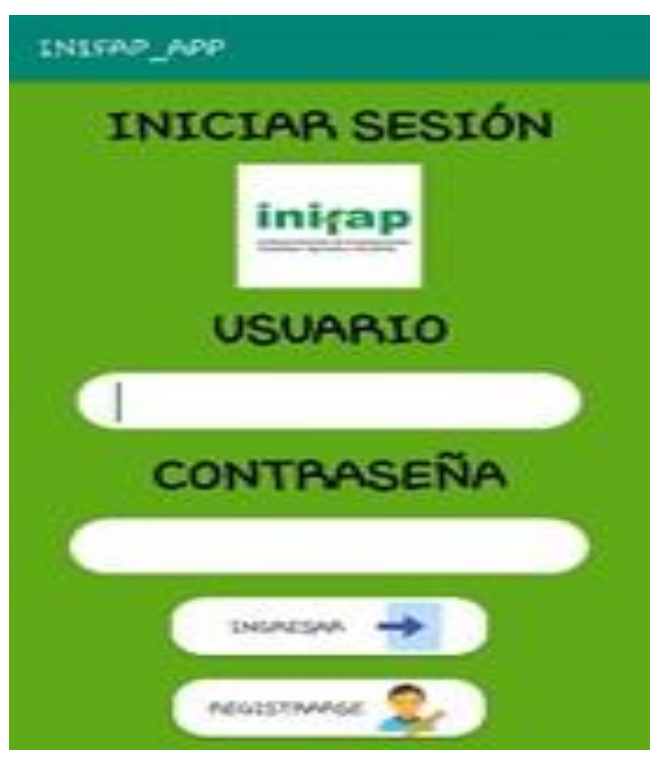

Figura 19 Vista iniciar sesión Fuente: Elaboración Propia

En caso de que, aún no sea un usuario registrado deberá hacer por primera vez el proceso de registrar sus datos, para que pueda acceder sin problema a las opciones que brinda la aplicación móvil, tal como se observa en la figura 20 .

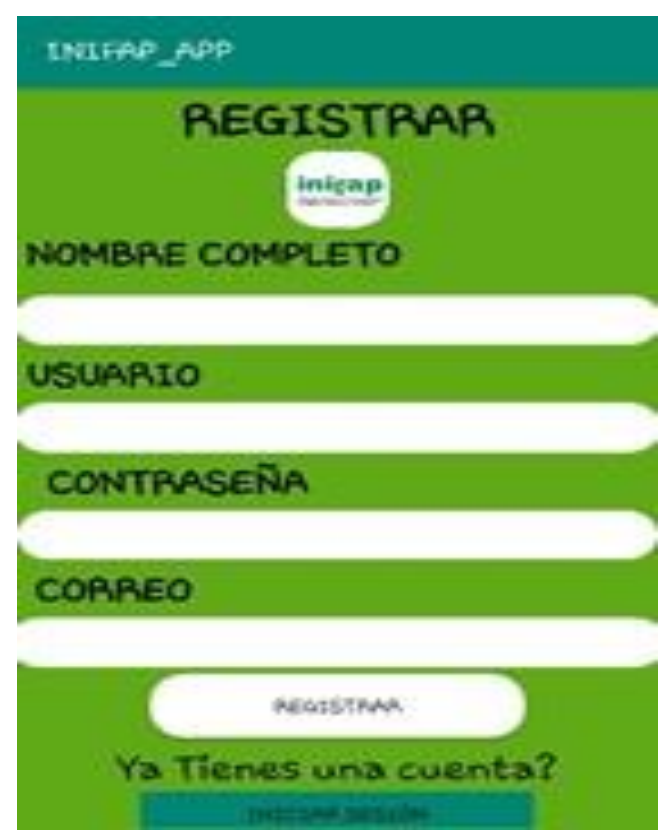

Figura 20 Vista registrar usuario Fuente: Elaboración Propia

En la siguiente figura se puede observar el menú principal una vez que se ha autentificado el usuario, para hacer uso de las opciones que brinda este menú, tales como: ¿Quiénes Somos?, Datos Climatológicos, Directorio, Publicaciones.

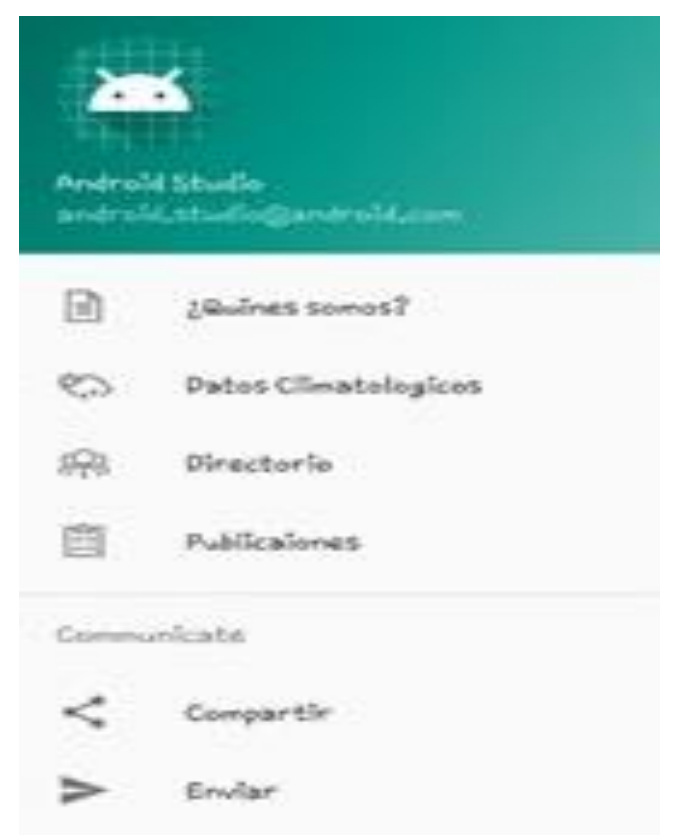

Figura 21 Vista menú principal Fuente: Elaboración Propia

Con este menú de opciones se pretende que la aplicación móvil brinde a los usuarios la misma experiencia como opera exactamente el sitio web con la diferencia de que desde esta aplicación será otra opción más para ingresar a los recursos tecnológicos que ofrezca el inifap a los productores de maíz en corto plazo.

DE LEÓN-CASTREJÓN, Andrés, MELQUIADES-JIMÉNEZ, José Uriel y NORIEGA-CANTÚ, David Heriberto. Sitio Web y aplicación móvil para el control de datos climatológicos a tráves de sensores en un prototipo de estación agrometeorológica. Revista de Cómputo Aplicado. 2019. 


\section{Agradecimiento}

Se hace extensivo un agradecimiento y reconocimiento muy especial al Dr. David Heriberto Noriega Cantú y al Ing. José Uriel Melquiades Jiménez que apoyaron en la realización de este trabajo en extenso.

\section{Conclusiones}

- Se construyó la base de datos eclima que incluye las tablas, tales como: contacto, directorio, estaciones, estacion_agrometeorologica, estados, login, municipios, posts, tbl_tokens, y usuario.

- Se diseñaron las interfaces gráficas de usuario, tales como: el login para sesiones de usuarios y administrador, datos climatológicos, estaciones, mapas, datos diarios, publicaciones, artículos científicos, folletos técnicos, directorio, inifap guerrero y email.

- $\quad$ Se desarrollaron los módulos en php para el control de información y procesamiento en el sitio web.

- Se diseñó una interfaz gráfica de usuario, mostrando un mapa, con ubicaciones específicas para actualizar, eliminar y agregar cada estación agrometeorológica posicionándolas en algún punto geográfico del estado de guerrero.

- Se diseñaron las interfaces gráficas para la aplicación móvil con las opciones para consultar los datos diarios e históricos de las variables climatológicas aun en diseño.

- Se contrató un hosting y se creó el dominio datoseclima.opds.website para alojar el sitio web y la base de datos del proyecto para realizar pruebas.

- Se realizó la comunicación del prototipo de estación agrometeorológica a través de la tarjeta de adquisición de datos xatellite y la plataforma ubidots.com por medio de su api web service.

\section{Trabajos futuros}

- Implementar dos api restful service para conectar la base de datos del sitio web con la tarjeta de adquisición de datos xatellite y otro para conectar la aplicación móvil con el sitio web de forma remota.
- $\quad$ Se reestructure el nombre del dominio para que sea adecuado al inifap y a la utrng, una vez terminadas las pruebas en el hosting.

- Reconstruir las estaciones agrometeorológicas que tienen fallas y darles mantenimiento para reactivarlas.

- Mejorar el sitio web para que esté en condiciones de soportar los datos de las variables climatológicas de las estaciones agrometeorológicas que se vayan reactivando sobre la marcha.

- Generar un id o api key y un token por cada estación agrometeorológica que sea registrada en el sitio web y desde la aplicación móvil.

\section{Referencias}

[1].Bueno, Solano, R. J., Morales, L., \& De Jesus, C. (2019). Diseño de una Estacion Meteorologica para el Laboratorio de Simulaciones Udes.

[2].Castro Mesa, J. F. (2019). Diseño e Implementación de un Sistema en la Nube para el Seguimiento y Monitoreo permanente de Variables Ambientales en Cultivos de Uva en el Valle del Cauca.

[3].Instituto Nacional de Investigaciones Forestales, A. y. (2015). INIFAP, Campo Experimental Iguala. Obtenido de http://www.inifap.gob.mx/SitePages/inifap2 015/Quienes_Somos/quienes_somos.aspx

[4].Jacobson I, B. (2000). El Proceso Unificado de Desarrollo de Software. España: Pearson Educacion Addison Wesley.

[5].Masanet, M. I., Capraro, F., Klenzi, R. O., Muñoz, M., \& Suárez, C. (June, 2019). Entorno Web de Visualización de Información Meteorológica para el Uso Agrícola y de Generación de Alertas ante Eventos Climáticos. In XXI Workshop de Investigadores en Ciencias de la Computación (WICC 2019, Universidad Nacional de San Juan).

[6].Mendez González, L. C. (2019). Prototipo de Estación Meteorológica. Instituto de Ingeniería y Tecnología. 
[7].Pacheco E., A. (2008). Metodología Crítica de la Investigación, Lógica, Procedimiento y Técnicas. México: Grupo Editorial Patria.

[8].Pressman Roger, S. (2005). Ingeniería de Software, Un Enfoque Práctico, Sexta Edición. México: Mc Graw-Hill.

[9].Rojas, D., \& Ernesto, P. (2019). Eficiencia del Servicio de Información Turística Oficial de la DIRCETUR Cusco para la Toma de Desiciones del Turista Receptivo 2017: Alternativa Aplicación Digital Móvil.

[10]. Vizcarra, M., Malory, J., \& Yshida Riva, C. H. (2019). Terminal Terrestre Sostenible con Aplicación de Energia Solar, para la Ciudad de Abancay. 